\title{
Risk factors for radiation induced lymphopenia in patients with breast cancer receiving adjuvant radiotherapy
}

\author{
Fang Chen ${ }^{1}$, Hao Yu ${ }^{2}$, Hong Zhang ${ }^{3}$, Yaqing Nong ${ }^{1}$, Qian Wang ${ }^{1}$, Haiman Jing ${ }^{1}$, Ying Han ${ }^{1}$, Junjie Wu ${ }^{1}$, \\ Zheng Zhou ${ }^{1}$, Li Yang ${ }^{1}$, Zhiyuan $\mathrm{Xu}^{1}$, Yaya Liu ${ }^{1}$, Pingfu Fu ${ }^{4}$, Jian-Yue Jin ${ }^{5}$, Victor Hsue ${ }^{1}$, Amy Chang $^{6}$, \\ Feng-Ming (Spring) Kong ${ }^{1,7}$
}

${ }^{1}$ Department of Clinical Oncology, The University of Hong Kong-Shenzhen Hospital, Shenzhen, China; ${ }^{2}$ Institute of Advanced Technology, Chinese Academy of Sciences, Shenzhen, China; ${ }^{3}$ Department of Radiation Oncology, University of Maryland School of Medicine, Baltimore, MD, USA; ${ }^{4}$ Department of Population and Quantitative Health Sciences, Case Western Reserve University, Cleveland, OH, USA; ${ }^{5}$ Department of Radiation Oncology, Case Western Reserve University, Cleveland, OH, USA; ${ }^{6}$ Comprehensive Oncology Center, Hong Kong Sanatorium \& Hospital, Hong Kong, China; ${ }^{7}$ Department of Clinical Oncology, Hong Kong University Li Ka Shing Medical School, Hong Kong, China

Contributions: (I) Conception and design: F Chen, JY Jin, FM Kong; (II) Administrative support: Z Xu, V Hsue, A Chang, FM Kong; (III) Provision of study materials or patients: F Chen, H Jing, L Yang; (IV) Collection and assembly of data: F Chen, Y Nong, Q Wang, Y Han, J Wu, Z Zhou, Y Liu; (V) Data analysis and interpretation: H Yu, H Zhang, P Fu, F Chen, Y Liu; (VI) Manuscript writing: All authors; (VII) Final approval of manuscript: All authors.

Correspondence to: Feng-Ming (Spring) Kong, MD, PhD, FACR, FAAWR, FASTRO. Department of Clinical Oncology, The University of Hong Kong-Shenzhen Hospital, Shenzhen, China; Department of Clinical Oncology, Hong Kong University Li Ka Shing Medical School, Hong Kong, China. Email: kong0001@hku.hk.

Background: This study aimed to investigate radiation-induced lymphopenia and its potential risk factors in patients with breast cancer receiving adjuvant radiotherapy.

Methods: Breast cancer patients received adjuvant radiotherapy (RT) at our hospital with peripheral lymphocyte counts (PLC) at pre-and immediately after RT (post-RT) were eligible. The primary endpoints were any grade of lymphopenia post-RT and nadir-PLC/pre-PLC $<0.8$. Patient characteristics, tumor factors, and treatment factors were collected for risk assessment. Data are presented as mean and $95 \%$ confidence interval (CI) unless otherwise specified. Matched analysis was used to compare the statistical significance between different RT techniques.

Results: A total of 735 consecutive patients met the study criteria. The mean PLC was $1.58 \times 10^{9} / \mathrm{L}$ before and $0.99 \times 10^{9} / \mathrm{L}$ post-RT $(\mathrm{P}<0.001)$. At the end of RT, $60.5 \%$ of patients had lymphopenia. Univariate and multivariable logistic analyses showed that RT technique involving RapidArc, mean lung dose, and chemotherapy were significant risk factors $(\mathrm{P}<0.05)$ for lymphopenia. RT technique was the only significant risk factor $(\mathrm{P}<0.05)$ for nadir-PLC/pre-PLC $<0.8$. Patients treated with RapidArc had a significantly greater reduction of PLC along with greater V5 of the lungs, even after matching mean lung dose and radiated volume.

Conclusions: Lymphopenia is common in patients with breast cancer after adjuvant RT. RT technique is the only significant factor for lymphopenia and nadir-PLC/pre-PLC $<0.8$, suggesting the significance of RT technique choice to minimize lymphopenia and improve treatment outcomes.

Keywords: Lymphopenia; risk factors; breast cancer; radiotherapy technique, RapidArc

Submitted Apr 26, 2021. Accepted for publication Jun 25, 2021.

doi: $10.21037 / \mathrm{atm}-21-2150$

View this article at: https://dx.doi.org/10.21037/atm-21-2150 


\section{Introduction}

According to the 2018 global cancer statistics report, breast cancer is the most common cancer in women and the world's second most common cancer (11.6\%) after lung cancer (1). Radiotherapy (RT) plays an important role in breast cancer treatment by reducing local tumor failure (2-4). At the same time, RT damages normal tissues including part of the immune system, subsequently results in impaired immune function and negatively impacts survival (5).

Baseline immune status is an important predictor for poor prognosis. Lymphopenia, or low peripheral lymphocyte count (PLC), predicts poor survival in patients with metastatic breast cancer $(6,7)$. In a cohort of 1,463 patients, Afghahi et al. also revealed that sufficiently higher PLC at diagnosis predicted lower mortality in earlystage triple-negative breast cancer (8).

Emerging evidence showing radiation-induced lymphopenia (RIL) or PLC-related parameters are associated with poor survival outcomes in several solid tumors (9-14). Cho et al. reported RIL was a potential predictor for ipsilateral breast tumor recurrence in 216 patients with early breast cancer treated with breast-conserving therapy (BCT) (15). Most recently, a post hoc analysis of 598 patients from a phase III randomized clinical trial reported significantly inferior 5 -year disease-free survival in patients with a nadir-PLC/pre-PLC ratio $<0.8$ in breast cancer patients treated with mastectomy followed by adjuvant RT (16). Another study reported that higher PLC was correlated with a higher treatment response rate in solid tumor patients who received checkpoint inhibitors (17).

However, it is unclear what the risk factors associated with RIL are. The above-mentioned phase III study reported potential risks, including a limited number of factors such as low body mass index (BMI), right-sided tumors, and the use of neoadjuvant chemotherapy (16). This study involved a larger patient cohort and aimed to investigate the risk factors for RIL, focusing on treatmentrelated risk factors, including radiation dosimetric factors and radiotherapy techniques.

We present the following article in accordance with the STROBE reporting checklist (available at https://dx.doi. org/10.21037/atm-21-2150).

\section{Methods}

\section{Study population}

All patients with breast cancer who had received adjuvant
RT between March 2015 to February 2020 at the University of Hong Kong-Shenzhen Hospital formed the original study population. Other eligibility criteria included: pathology-confirmed invasive breast cancer, aged 18 years and above, and PLCs within 7 days before the start of RT (pre-RT) and 7 days from the completion of RT (postRT) at this same hospital. Exclusion criteria were patients received RT for non-invasive breast cancer (stage 0), stage IV, or recurrent breast cancer; breast lymphoma; and patients with immune-related diseases. Breast cancer patients who underwent surgery and/or chemotherapy in other hospitals were eligible if they received radiation at this hospital.

Complete blood counts (CBC) with the inclusion of lymphocyte counts were tested per hospital standard practice within 7 days before, during, and at the end of RT, as well as $1-3$ months after RT. Radiotherapy techniques employed included the 2 fields tangential opposing technique (2D-field), 3-D conformal technique (3DCRT), and RapidArc (Varian Medical Systems, Palo Alto, CA, USA). CT scans from the skull base to the level of the first lumbar vertebra were obtained for all patients in this study. The 2D-field technique was employed on patients who needed irradiation for the breast only, following BCT. 3DCRT was employed on patients who needed irradiation of the breast or chest wall and supraclavicular (SCF) lymph node and/or axillary fossa and excluded the internal mammary node (IMN). IMN irradiation was applied in patients with invasive breast cancer with $\mathrm{N} 3$ or N2 diseases with centrally or medially located primary tumors. RapidArc, a volume modulated arc therapy (VMAT) technique, was employed on patients who needed irradiation of the IMN. The clinical target volume of this RT included the breast or chest wall and the locoregional lymphatics, which were treated using 2 partial arcs. For the left breast, 2 arcs from $\sim 160^{\circ}$ to $300^{\circ}$ were used ( 1 arc was clockwise, the other counterclockwise). For the right breast, 2 arcs from $\sim 200^{\circ}$ to $60^{\circ}$ were used ( 1 arc was clockwise, the other counterclockwise). Dose limits to the organs at risk included the following: V5 of ipsilateral lung 65/70, V10 of ipsilateral lung 50/60, V20 of ipsilateral lung 30/35, and mean heart dose 4 Gy.

\section{Data collection}

The following variables were collected retrospectively: (I) patient factors including age and menstrual status; (II) tumor factors including tumor laterality, clinical TNM 
stage, and ER/PR/HER-2 subtype; (III) non-radiation treatment factors, including surgical approach, prior chemotherapy, chemotherapy regimen, endocrine therapy, and anti-HER2 target therapy; (IV) radiation factors, including RT technique, number of treatment fields, fractionation, mean heart dose, mean dose of ipsilateral/ bilateral lungs, and total treatment volume; and (V) PLC, granulocytes, monocytes, hemoglobin, and platelets in CBC. Nadir-PLC was defined as the lowest PLC, and prePLC was defined as PLC within 1 week before the start of RT. Grade 1, 2, 3, and 4 lymphopenias were defined using PLC cut-off of LLN (lower limits of normal) $-0.8 \times 10^{9}$, $(0.8-0.5) \times 10^{9},(0.5-0.2) \times 10^{9}$, and $0.2 \times 10^{9} / \mathrm{L}$, respectively, following the Common Terminology Criteria for Adverse Events (CTCAE) version 5.0. Lymphopenia was defined using PLC cut-off of LLN, which was $1.06 \times 10^{9} / \mathrm{L}$ in our institution. Additionally, the relative value of nadir-PLC/ pre-PLC $<0.8$ was also considered radiation-induced lymphopenia as it was associated with inferior 5 -year disease-free survival (DFS) in a study by Sun et al. (16).

The study was conducted in accordance with the Declaration of Helsinki (as revised in 2013). The study was approved by the ethics committee of the University of Hong Kong-Shenzhen Hospital (\# 2019 098), and individual consent for this retrospective analysis was waived.

\section{Statistical analysis}

The primary endpoints of the study were: (I) lymphopenia post-RT, which was assessed within 7 days from the end of RT, defined as above by CTCAE; and (II) nadir-PLC/ pre-PLC, which was defined as the ratio of lowest PLC during radiation and PLC before radiation. The predictive variables of interest include patient factors such as age and menopausal status, tumor features such as stage and ER/PR/HER-2 subtype, as well as treatment-related parameters such as chemotherapy, surgical approach, and radiation dosimetric factors. The effects of potential risk factors were estimated using univariate logistic analysis initially. To avoid the unstable and inaccurate estimates of the coefficients, multicollinearity was tested, and those that were significant in univariate analysis were further evaluated using a linear cross-correlation matrix. Pearson's coefficient was used to evaluate the association between continuous variables. The Spearman correlation was used to evaluate the association between categorical variables. Some highly correlated dosimetric variables, such as mean heart dose, were excluded in the final multivariable logistic regression due to multicollinearity. Data are presented as mean $[95 \%$ confidence interval (CI)] unless otherwise specified. Paired $t$-test was used to examine the difference in continuous measurement pre- and post-radiation therapy. $\mathrm{P}$ values less than 0.05 were considered to be statistically significant. Patients treated with RapidArc were more likely to have a greater volume of irradiated lung and treatment volume, and thus, matched analysis with mean lung dose and radiation treatment volume was performed to evaluate the influence of RapidArc versus 3DCRT for a more balanced comparison. The match analysis matched specific variables (such as mean lung dose) by pairing each patient in the RapidArc group with a patient in the 3DCRT group who had the smallest difference in terms of the variables. Several pairs of patients with similar specific variables were identified, and thus other variables could be compared without the interference of the specific variables.

\section{Results}

\section{Characteristics of the patient, tumor, and prior systemic treatments}

Between March 2015 to February 2020, a total of 735 patients met the study criteria (Figure 1). Table 1 lists the patient's characteristics, tumor, pre-radiation treatment factors at baseline, and radiation dosimetric factors. The median age of patients was 42 years, with the majority being premenopausal (75.5\%). A total of 456 patients $(62.3 \%)$ received regional nodal RT, and the remaining $37.7 \%$ of patients received breast RT alone for BCT. A total of 666 patients (90.6\%) received chemotherapy before adjuvant RT, and $24.4 \%$ of patients received anti-HER2 target therapy before and concurrent with adjuvant RT. The majority of patients received RT by 2D-field technique or 3DCRT, while $16.7 \%$ of patients received RT by RapidArc. The vast majority of patients $(90.5 \%)$ received hypofractionated irradiation (40.05 Gy in 15 fractions in 3 weeks).

\section{PLC at the beginning and post-RT}

As shown in Table 2, prior to RT commencement, the mean PLC was $1.58 \times 10^{9} / \mathrm{L}\left(95 \%\right.$ CI: $\left.1.54 \times 10^{9}-1.62 \times 10^{9} / \mathrm{L}\right)$ and $14.3 \%$ (105/735, 95\% CI: $14.2-14.3 \%)$ of patients had CTCAE-defined lymphopenia: $11.4 \%, 2.5 \%, 0.4 \%$, and $0 \%$ for grade $1,2,3$, and 4 , respectively.

After commencement of RT, the mean PLC was $0.99 \times 10^{9} / \mathrm{L}\left(95 \%\right.$ CI: $\left.0.96 \times 10^{9}-1.01 \times 10^{9} / \mathrm{L}\right)$, which was 


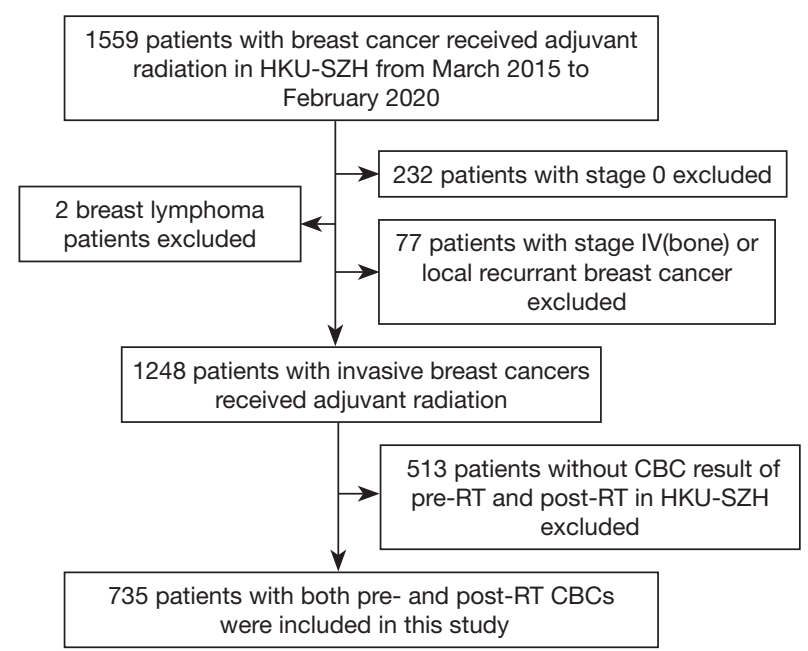

Figure 1 Study population profile. As shown, 1,559 patients with breast cancer received adjuvant radiotherapy (RT) between March 2015 to February 2020 at the University of Hong Kong-Shenzhen Hospital, and a total of 735 patients met the study criteria for inclusion.

Table 1 Patient characteristics at baseline (N=735)

\begin{tabular}{lc}
\hline Patient characteristics & No. of patients (\%) \\
\hline Age & $45(26-86)$ \\
Median (range), year & \\
Menopausal status & $555(75.5)$ \\
Premenopausal & $180(24.5)$ \\
Postmenopausal & \\
Tumor laterality & $371(50.5)$ \\
Left & $363(49.4)$ \\
Right & $1(0.1)$ \\
Bilateral & \\
Modified N stage & \\
No & $290(39.5)$ \\
N+ & $445(60.5)$ \\
Modified stage & \\
I (IA/IB) & $193(26.2)$ \\
II (IIA/IIB) & $332(45.2)$ \\
III (IIIA/IIIB/IIIC) & $210(28.6)$ \\
\hline
\end{tabular}

Table 1 (continued)
Table 1 (continued)

\begin{tabular}{|c|c|}
\hline Patient characteristics & No. of patients (\%) \\
\hline \multicolumn{2}{|l|}{ Immunohistochemistry subgroup } \\
\hline HR+/HER2- & $436(59.3)$ \\
\hline HER2+/HR- & $77(10.5)$ \\
\hline HER2+/HR+ & $108(14.7)$ \\
\hline HR-/HER2- & $114(15.5)$ \\
\hline \multicolumn{2}{|l|}{ Surgical approaches } \\
\hline Breast conserving therapy $(\mathrm{BCT})$ & $373(50.7)$ \\
\hline Mastectomy & $362(49.3)$ \\
\hline Sentinel lymph node biopsy (SLNB) & $265(36.1)$ \\
\hline Axillary lymph node dissection (ALND) & $470(63.9)$ \\
\hline \multicolumn{2}{|l|}{ Chemotherapy strategy } \\
\hline None & $69(9.4)$ \\
\hline Neoadjuvant & $131(17.8)$ \\
\hline Adjuvant & $513(69.8)$ \\
\hline Neoadjuvant + adjuvant & $22(3.0)$ \\
\hline \multicolumn{2}{|l|}{ Chemotherapy regimens } \\
\hline None & $69(9.4)$ \\
\hline Anthracycline & $17(2.3)$ \\
\hline Taxane & $183(24.9)$ \\
\hline Anthracycline + taxane & $464(63.1)$ \\
\hline Others & $2(0.3)$ \\
\hline \multicolumn{2}{|l|}{ Anti-HER2 therapy } \\
\hline None & $556(75.6)$ \\
\hline Yes & $179(24.4)$ \\
\hline \multicolumn{2}{|l|}{ Endocrine therapy } \\
\hline None & $186(25.3)$ \\
\hline Yes & $549(74.7)$ \\
\hline \multicolumn{2}{|l|}{ Radiotherapy technique } \\
\hline RapidArc & $123(16.7)$ \\
\hline 2D-fields & $277(37.7)$ \\
\hline 3DCRT & $335(45.6)$ \\
\hline
\end{tabular}

Table 1 (continued) 
Table 1 (continued)

\begin{tabular}{lc}
\hline Patient characteristics & No. of patients (\%) \\
\hline RT fields & $277(37.7)$ \\
Tangential breast only & $322(43.8)$ \\
Breast/chest wall + SCF & $123(16.7)$ \\
Breast/chest wall + SCF + IMN & $13(1.8)$ \\
Breast/chest wall + SCF + Axillary & \\
RT dose and fractions & $665(90.5)$ \\
40.5 Gy/15 fx & $61(8.3)$ \\
50 Gy/25 fx & $9(1.2)$ \\
$50-60$ Gy/25 fx & \\
Use of RPM & $721(98.1)$ \\
None & $14(1.9)$ \\
Yes & $1,690.8$ \\
Treatment volume (95\% Cl), cc & $(1,652.2,1,729.4)$ \\
Mean heart dose (95\% Cl), Gy & $2.4(2.2-2.5)$ \\
Integral dose of the total body (95\% Cl), Gy & $4.4(4.3-4.5)$ \\
Mean dose of ipsilateral lung (95\% Cl), Gy & $10.1(9.8-10.3)$ \\
Mean dose of bilateral lungs $(95 \% \mathrm{Cl}), \mathrm{Gy}$ & $5.5(5.3-5.6)$ \\
\hline
\end{tabular}

${ }^{\dagger}$ Modified $\mathrm{N}$ stage: the higher $\mathrm{N}$ stage between clinic $\mathrm{N}$ stage and yp $\mathrm{N}$ stage for patients who had received neoadjuvant chemotherapy; ${ }^{\ddagger}$ modified stage: the higher stage between clinic stage and yp stage for patients who had received neoadjuvant chemotherapy. RT, radiotherapy; N, lymph nodes; BCT, breastconserving therapy; SLNB, sentinel lymph node biopsy; ALND, axillary lymph node dissection; SCF, supraclavicular lymph nodes; IMN, internal mammary nodal; fx, fraction; RPM, realtime position management.

significantly reduced compared to pre-RT $(\mathrm{P}<0.001)$ (Table 2). There were also significant reductions in other peripheral blood cells, including total white cell counts (WBC), neutrophil, platelet, and monocyte counts $(\mathrm{P}<0.001)$, excluding hemoglobin. The reduction of PLC was most remarkable and at the lowest PLC (nadir-PLC) near the end of radiation (Figure 2). All patients had preand post-RT data. At post-RT, $60.5 \%$ (445/735, 95\% CI: $60.4-60.6 \%)$ of patients had lymphopenia, with $30.7 \%$, $23.4 \%, 6.1 \%$, and $0.3 \%$ for grade $1,2,3$, and 4 , respectively (Table 2). $92.7 \%$ of patients had some degree of reduction in PLC, and $77.8 \%$ had nadir-PLC/pre-PLC $<0.8$. Of the $171(23.2 \%)$ patients for whom data was available for PLC 1-3 months after radiation in our hospital, 24\% (41/171) had persistent lymphopenia. Like PLC post-RT, PLC 1-3 months after RT was significantly lower than PLC preRT $(\mathrm{P}<0.001)$ (Figure 2).

\section{Risk factors of radiation-induced lymphopenia}

Results of univariate and multivariable logistic analyses are shown in Tables 3,4. The post-RT lymphopenia was significantly correlated with many factors, including tumor laterality, $\mathrm{N}$ stage, surgical approach, chemotherapy treatment, mean heart dose, mean ipsilateral and bilateral lung dose, mean integral dose of the total body, number of RT fields, RT fractionation schema, and RT volume (all $\mathrm{P}<0.05)$. Whereas age, menopausal status, ER/PR/HER-2 subgroup, endocrine therapy, and target therapy were not significant. The highly correlated dosimetric variables such as mean heart dose, chemotherapy regimen, and modified $\mathrm{N}$ stage were not included in the final multivariate logistic regression. Under multivariable analysis, only RT technique, mean lung dose, and chemotherapy treatment remained significant, while RT fractionation schema and the breast surgical approach did not. For nadir-PLC/pre$\mathrm{PLC}<0.8$, surgical approach and RT factors including RT technique, number of fields, mean ipsilateral and bilateral lung doses, and RT fractionation schema were significant factors, while chemotherapy treatment, age, menopausal status, tumor laterality, ER/PR/HER-2 subgroup, use of endocrine, and anti-HER2 therapy were not. Further multivariable logistic analyses showed radiotherapy technique was the only significant risk factor, while RT fractionation schema, mean lung dose, and the breast surgical approach was not. Radiotherapy technique was the only independent significant factor for both post-RT lymphopenia and nadir-PLC/pre-PLC $<0.8$.

\section{Radiation-induced lymphopenia and RT technique}

To understand the effect of the RT technique, additional analysis was performed in patients treated for the localregional disease. Patients with 2 -field RT were excluded from this analysis as their treatment volumes were remarkably different from this group. Patients treated with RapidArc had significantly greater PLC reduction 
Table 2 Complete blood counts (CBC) at the beginning and post-RT

\begin{tabular}{|c|c|c|c|}
\hline Blood testing parameter & Pre-RT & Post-RT & $P$ value \\
\hline Lymphocyte percent (LYM/WBC) (95\% Cl), \% & $29.7(29.1-30.4)$ & $23.2(22.7-23.7)$ & $<0.001$ \\
\hline Mean neutrophil (95\% Cl), $10^{9} / \mathrm{L}$ & $3.63(3.46-3.80)$ & $2.96(2.88-3.05)$ & $<0.001$ \\
\hline Mean WBC $(95 \% \mathrm{Cl}), 10^{9} / \mathrm{L}$ & $5.69(5.50-5.88)$ & $4.38(4.29-4.48)$ & $<0.001$ \\
\hline Mean hemoglobin $(95 \% \mathrm{Cl}), \mathrm{g} / \mathrm{L}$ & $117.7(116.8-118.6)$ & $122.6(121.8-123.5)$ & $<0.001$ \\
\hline Mean platelet $(95 \% \mathrm{Cl}), 10^{12} / \mathrm{L}$ & $239(235-244)$ & $196(192-199)$ & $<0.001$ \\
\hline Lymphopenia, n (\%) & $105(14.3)$ & $445(60.5)$ & $<0.001$ \\
\hline Grade 1 & $84(11.4)$ & $226(30.7)$ & \\
\hline Grade 4 & 0 & $2(0.3)$ & \\
\hline
\end{tabular}

${ }^{*}, \mathrm{P}$ values were assessed by matched $t$-test.

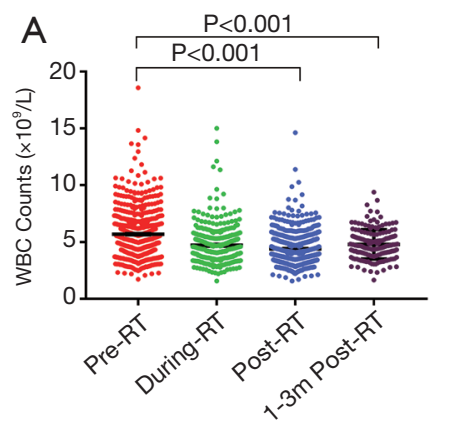

B

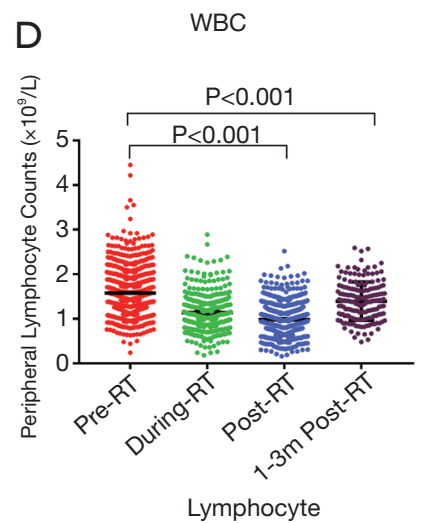

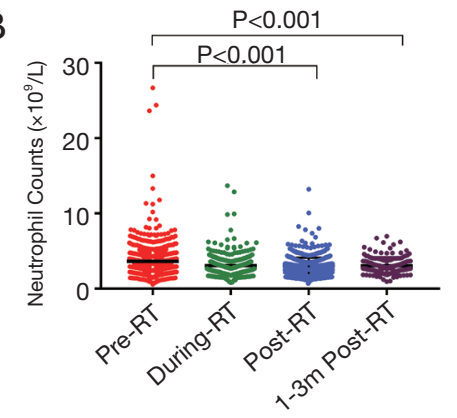

E

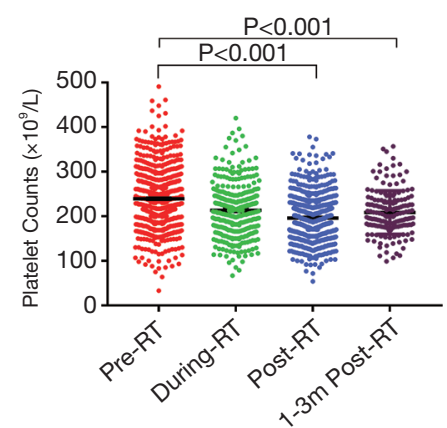

Platelet

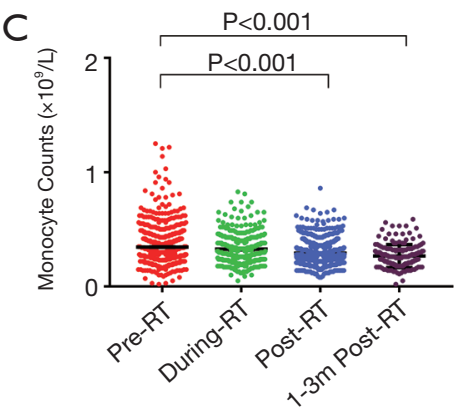

F

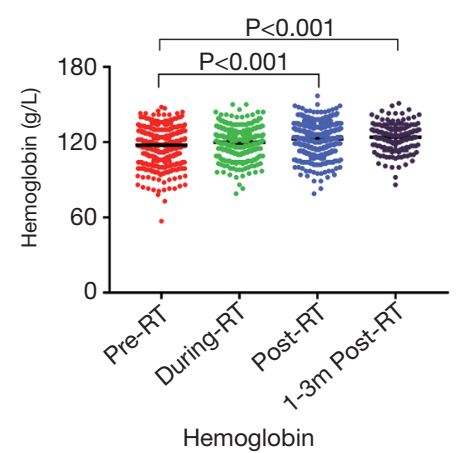

Figure 2 Change of different blood counts during radiotherapy (RT). The figure shows the dynamics of (A) total white blood cell (WBC), (B) neutrophil, (C) monocyte, (D) lymphocyte, and (E) platelet counts; and (F) hemoglobin before, during, and after radiation (pre-RT, duringRT, immediately post-RT, and 1-3 months after RT). There were significant reductions in the level of peripheral lymphocyte counts (PLC), total white blood cell, neutrophil, platelet, and monocyte counts $(\mathrm{P}<0.001)$, while hemoglobin increased. The reduction of $\mathrm{PLC}$ was the most obvious among these peripheral blood cells in complete blood counts (CBC) after radiation. The lowest PLC (nadir-PLC) was at the end of radiation (post-RT). 
Table 3 Univariate logistic analysis of lymphopenia post-RT and nadir-PLC/pre-PLC $<0.8$

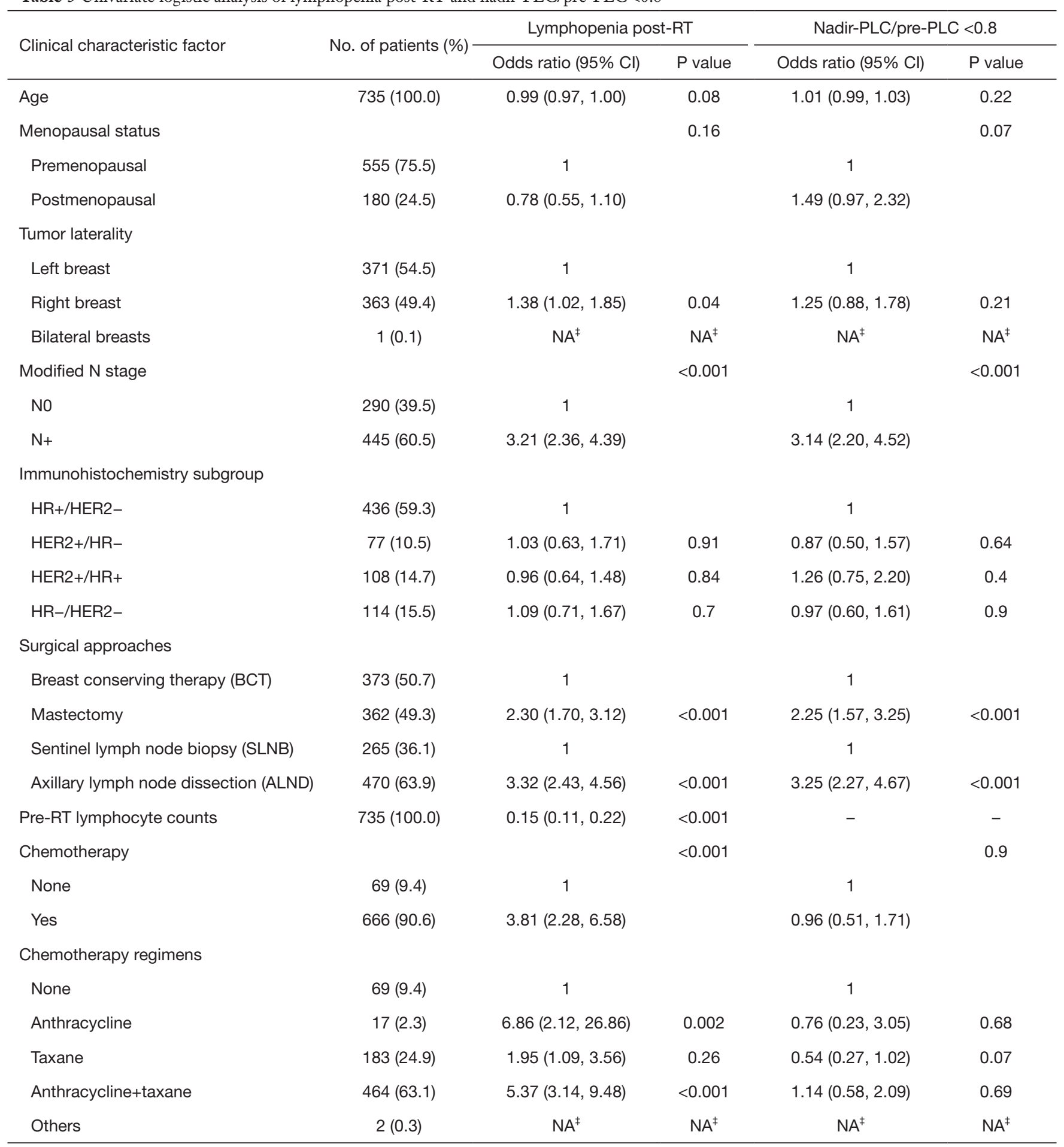

Table 3 (continued) 
Table 3 (continued)

\begin{tabular}{|c|c|c|c|c|c|}
\hline Clinical characteristic factor & No. of patients (\%) & \multicolumn{2}{|c|}{ Lymphopenia post-RT } & \multicolumn{2}{|c|}{ Nadir-PLC/pre-PLC $<0.8$} \\
\hline \multicolumn{6}{|l|}{ Chemotherapy strategy } \\
\hline None & $69(9.4)$ & 1 & & & \\
\hline Neoadjuvant & $131(17.8)$ & $7.35(3.89,14.30)$ & $<0.001$ & $4.83(1.93,13.27)$ & 0.001 \\
\hline Neoadjuvant + adjuvant & $22(3.0)$ & $3.82(1.43,10.85)$ & 0.01 & $0.93(0.31,3.18)$ & 0.90 \\
\hline Anti-HER2 therapy & & & 0.91 & & 0.76 \\
\hline None & $556(75.6)$ & 1 & & 1 & \\
\hline Yes & $179(24.4)$ & $1.02(0.72,1.44)$ & & $1.07(0.71,1.62)$ & \\
\hline Yes & $549(74.7)$ & $0.94(0.66,1.32)$ & & $1.17(0.79,1.72)$ & \\
\hline \multicolumn{6}{|l|}{ Radiotherapy technique } \\
\hline RapidArc & $123(16.7)$ & 1 & & 1 & \\
\hline 2D-fields & $277(37.7)$ & $0.04(0.02,0.09)$ & $<0.001$ & $0.03(0.01,0.09)$ & $<0.001$ \\
\hline 3DCRT & $335(45.6)$ & $0.11(0.05,0.22)$ & $<0.001$ & $0.08(0.01,0.26)$ & $<0.001$ \\
\hline RT fields & & & $<0.001$ & & $<0.001$ \\
\hline Breast & $277(37.7)$ & 1 & & 1 & \\
\hline Breast/chest wall + regional $\mathrm{LNs}^{\dagger}$ & $458(62.3)$ & $3.64(2.67,5.00)$ & & $3.80(2.65,5.50)$ & \\
\hline $15 \mathrm{fx}$ & $665(90.5)$ & 1 & & 1 & \\
\hline $25 \mathrm{fx}$ & $70(9.5)$ & $3.13(1.73,6.07)$ & & $2.34(1.16,5.38)$ & \\
\hline Use of RPM & & & 0.07 & & 0.49 \\
\hline None & $721(98.1)$ & 1 & & 1 & \\
\hline Yes & $14(1.9)$ & $3.99(1.08,25.77)$ & & $1.71(0.46,11.07)$ & \\
\hline Treatment volume, cc & $735(100.0)$ & $1.001(1.001,1.002)$ & $<0.001$ & $1.002(1.001,1.002)$ & $<0.001$ \\
\hline Mean heart dose, Gy & $735(100.0)$ & $1.24(1.15,1.35)$ & $<0.001$ & $1.25(1.13,1.39)$ & $<0.001$ \\
\hline Integral dose of the total body, Gy & $735(100.0)$ & $1.99(1.73,2.32)$ & $<0.001$ & $2.03(1.69,2.48)$ & $<0.001$ \\
\hline Mean dose of bilateral lungs, Gy & $735(100.0)$ & $1.51(1.39,1.66)$ & $<0.001$ & $1.46(1.32,1.62)$ & $<0.001$ \\
\hline
\end{tabular}

${ }^{\dagger}$ Regional LNs: include supraclavicular, axillary levels I-III, and internal mammary nodal; NA ${ }^{\ddagger}$ : sample was too small to analyze. RT, radiotherapy; N, lymph nodes; BCT, breast-conserving therapy; SLNB, sentinel lymph node biopsy; ALND, axillary lymph node dissection; fx, fraction; RPM, real-time position management. 
Table 4 Multivariable regression analysis of risk factors for lymphopenia post-RT and nadir-PLC/pre-PLC $<0.8$

\begin{tabular}{|c|c|c|c|c|c|}
\hline Clinic characteristic factor & No. of patients (\%) & \multicolumn{2}{|c|}{ Lymphopenia post-RT } & \multicolumn{2}{|c|}{ Nadir-PLC/pre-PLC $<0.8$} \\
\hline Surgical approaches & & & 0.23 & & 0.18 \\
\hline Breast conserving therapy & $373(50.7)$ & 1 & & 1 & \\
\hline Mastectomy & 362 (49.3) & $0.76(0.48,1.19)$ & & $0.68(0.39,1.18)$ & \\
\hline None & $69(9.4)$ & 1 & & - & - \\
\hline Yes & $666(90.6)$ & $2.31(1.30,4.24)$ & & - & - \\
\hline \multicolumn{6}{|l|}{ Radiotherapy technique } \\
\hline RapidArc & $123(16.7)$ & 1 & & 1 & \\
\hline $\mathrm{RT}$ fractions & & & 0.41 & & 0.98 \\
\hline $15 \mathrm{fx}$ & $665(90.5)$ & 1 & & 1 & \\
\hline $25 \mathrm{fx}$ & $70(9.5)$ & $1.34(0.68,2.80)$ & & $1.01(0.45,2.49)$ & \\
\hline Mean dose of bilateral lungs, Gy & $735(100.0)$ & $1.23(1.07,1.42)$ & 0.003 & $1.13(0.97,1.34)$ & 0.13 \\
\hline
\end{tabular}

than 3DCRT, while WBC, neutrophil, and monocyte counts were not significantly different between these two groups (Figure 3). There was no significant difference in age or prior chemotherapy between these 2 groups. Most patients received chemotherapy before RT (96.7\% in the RapidArc group and $98.8 \%$ in the 3DCRT group). Hypofractionated irradiation was employed in $78 \%$ of patients in the RapidArc group and $88 \%$ in the 3DCRT group. RT treatment volumes and mean lung doses, and fractionation were significant factors for RIL, and thus analysis with matches for these factors was performed. This demonstrated that the RapidArc group had greater RIL (lower post-RT lymphocyte and lower nadir-PLC/pre-PLC ratio) than 3 DCRT $(\mathrm{P}<0.05)$. In these matched groups, patients treated with RapidArc still had greater PLC reduction, along with significantly lower V20s and higher V5s of the lungs (Figure 4).

\section{Discussion}

Our study of 735 patients with invasive breast cancer demonstrated a significant risk of lymphopenia after adjuvant RT. At the end of RT, $60.5 \%$ of patients had CTCAE-defined lymphopenia, $92.7 \%$ of patients had some degree of reduction in PLC, and $77.8 \%$ had nadirPLC/pre-PLC $<0.8$. The reduction of PLC was most remarkable in the reductions of all types of peripheral blood cells. While many factors were identified as risk factors for RIL, this study found that radiation dosimetric factors (such as RT technique and mean lung dose) were the most significant risk factors for RIL, and RT technique was the only risk factor for nadir-PLC/pre-PLC $<0.8$. RapidArc technique was a significant independent risk factor for both RIL and nadir-PLC/pre-PLC $<0.8$, even after volume and fractionation-matched analysis. RapidArc was associated with greater PLC reduction and greater V5s, but lower V20s of the bilateral lungs.

This is likely the first and the largest study examining risk factors for RIL in breast cancer patients, including radiation technique and dosimetric factors for lymphopenia and clinical significance. Our findings are consistent with the previous reports of several smaller studies $(15,16)$. An early observational study of 34 patients by Stjernswärd et al. in 1972 (18) reported long-term changes in PLC induced by RT in patients with breast cancer and their relation to tumor responses. Another small study (14 patients) performed by Standish et al. also noted this trend of changes after radiation (19). Our study of 

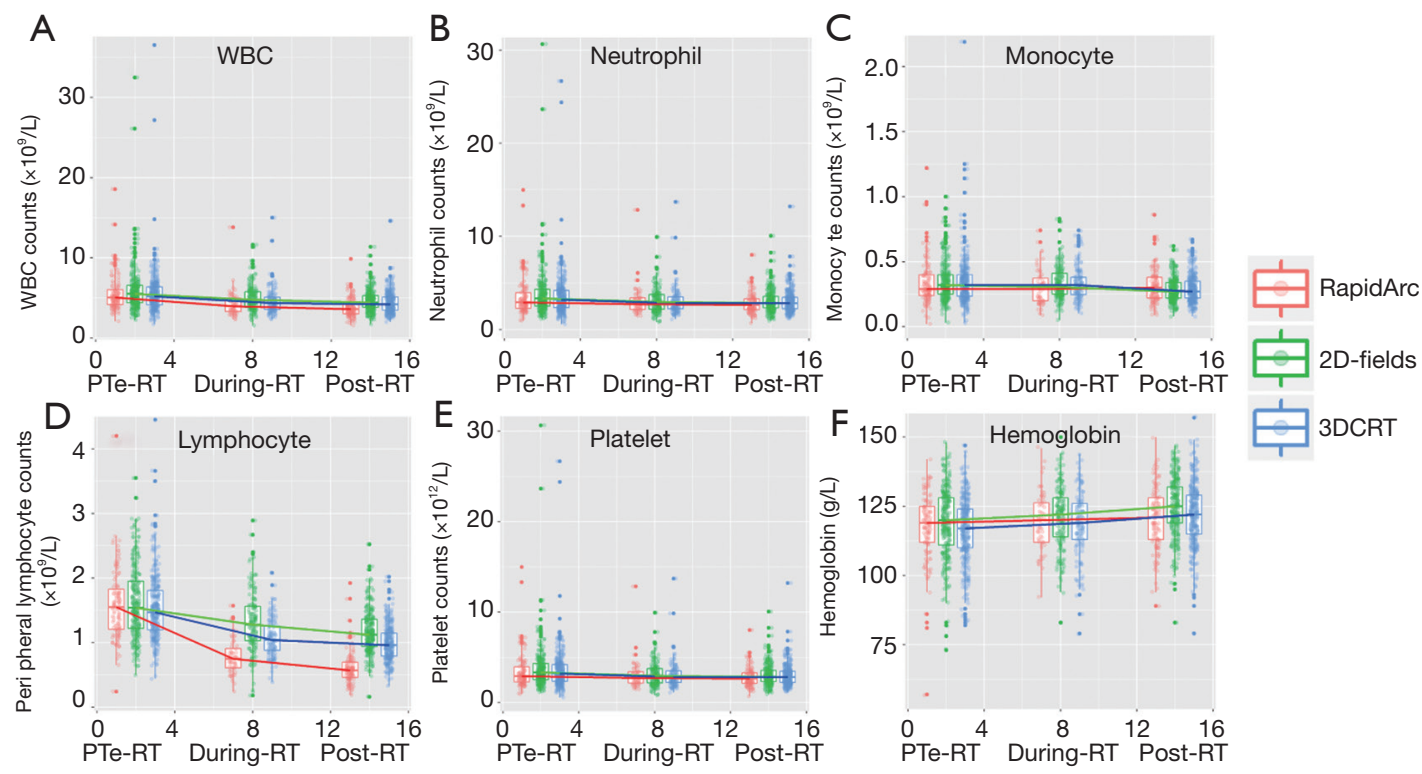

Figure 3 Changes in different blood cell counts and radiotherapy (RT) techniques. This figure shows changes of (A) WBC, (B) neutrophil, (C) monocyte, (D) lymphocyte, and (E) platelet counts, and (F) hemoglobin during RT, for 3 RT technique groups. Patients treated with RapidArc had a more significant reduction in peripheral lymphocyte counts (PLC) than 3D conformal radiation therapy (3DCRT), while counts of WBC, neutrophil, and monocyte were not significantly different between these 2 groups.

A
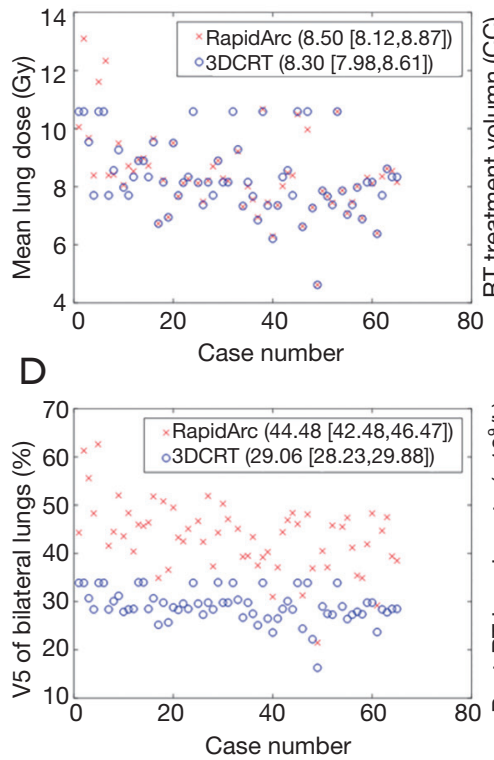

B

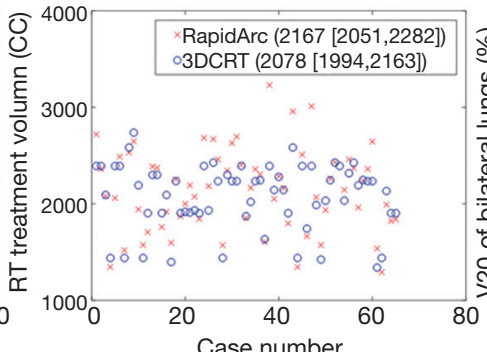

E

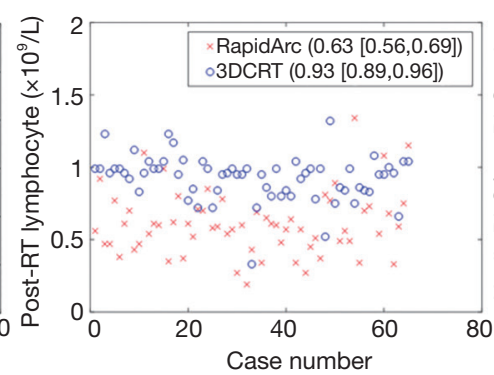

C

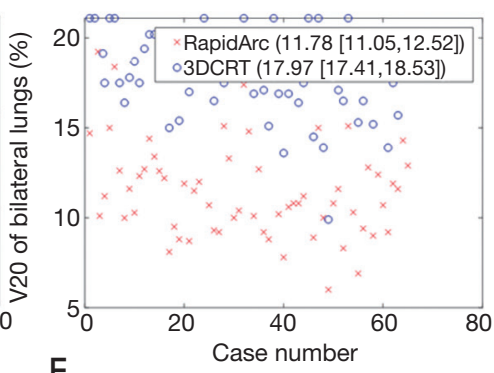

$\mathrm{F}$

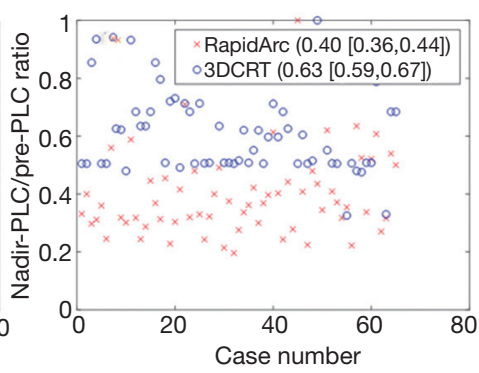

Figure 4 Radiation-induced lymphopenia and radiotherapy (RT) technique: RapidArc compared with 3D conformal radiation therapy (3DCRT). When mean bilateral lung doses (A), radiation treatment volume (B), and RT fractionation were matched, patients treated with RapidArc had significant lower V20 of bilateral lungs (C), higher V5 of bilateral lungs (D), significantly lower post-RT lymphocyte (E), and lower nadir-PLC/pre-PLC ratio (F), compared to patients treated with 3DCRT. 
735 patients demonstrated frequent RIL and heterogeneous reduction in lymphocytes after adjuvant radiotherapy. These findings have important clinical implications as a reduction in PLC is associated with immunosuppression, which significantly affects local control and survival in patients with breast cancer, as reported in several studies $(6-8,15,16)$.

Our study focused on risk factors, particularly treatment factors such as RT technique and dosimetry. The significance of dosimetric factors on RIL shown in this study was consistent with the findings of Tang et al. in lung cancer (10) and Ellsworth et al. in gastrointestinal cancers (20). Lymphocytes are radiosensitive, and exposure to even 2 Gy of radiation can cause lymphocyte cell death because they cannot repair DNA $(21,22)$. High-dose radiotherapy directly damages the lymphoid tissue within the radiation fields. RIL is believed to be caused by direct cell killing of circulating lymphocytes as they pass through the radiated field in thoracic cancers and immune organs like lymph nodes. Saito et al. reported that whole-body low-dose irradiation volume was associated with grade 3 or higher RIL in patients undergoing palliative RT (23). The significance of dosimetric correlations suggests that circulating lymphocytes may be considered an organ at risk (OAR) during RT planning $(20,24)$. More detailed modeling of radiation to lymphocytes as an immunosuppression model is ongoing in our group.

It is interesting to note that RapidArc was associated with more RIL compared to the 3DCRT technique. One possible explanation is the increased lung dose and enlarged treatment volume with more advanced diseases. However, after matching mean lung doses, RT treatment volume, and RT fractionation, the RapidArc group still had a higher incidence of RIL. Interestingly, patients in the RapidArc group had significantly higher V5 but lower V20 of the bilateral lungs. This V5 significance is a new finding for breast cancer patients and is consistent with the findings of Tang et al. that V5 of the lungs is a significant dosimetric predictor of post-RT lymphocyte nadir in patients with lung cancer (10). Similar findings from studies on different cancers also strongly implicate the effect of normal tissue damage due to low-dose radiation. A significant relationship with V5 instead of V20 confirms the importance of low dose effect, which has a biological rationale and suggests a potential role of radiation-sensitive circulating lymphocytes.

It should be noted that although patients treated with RapidArc often needed IMN coverage, and that there were more RIL in these patients, we did not intend to study the controversial benefit of IMN radiation. However, this study demonstrated that the use of RapidArc with irradiation to the IMN, despite its advantage on target coverage and meeting organ dose limits, did generate greater volumes of low dose radiation, which was associated with more RIL. This finding suggests a need for consideration of RIL as a detrimental factor in selecting patients to be treated by RapidArc in order to irradiate the IMN, particularly for patients with lower recurrence risk, such as lateral located N1 patients with HR+/HER2- subtype. In addition, RapidArc, a new and more expensive technique, can cause more RIL than 3DCRT, which may negatively impact survival in breast cancer patients. Should advanced radiotherapy techniques such as RapidArc,VMAT and tomotherapy be used to achieve the goals of target coverage or other conventional-dose limits, cautions should also be placed to limit the volume of low radiation, such as V5 of the lungs. While further study is needed to explore the full influence of radiation technique or dosimetric factor on other organs, findings from this study suggest that the use of RapidArc should be well justified for indications and carefully planned to decrease low dose volumes like V5.

Fractionation may be associated with RIL. Under univariate analysis, our study revealed that the hypofractionation group (15 fx) had less RIL compared to conventional fractionation $(25 \mathrm{fx})$. This finding is consistent with findings from Crocenzi et al. that hypofractionated RT was associated with significantly higher post-RT PLC (less RIL) and higher populations of major lymphocyte subpopulations (including CD4 and CD8 cells) compared with conventionally fractionated radiation in pancreatic cancer (25). Similarly, for breast cancer, Yuan et al. found that PLC post-RT in the hypofractionation group (13 fx) was higher (less RIL) than the conventional fraction group in a comparative analysis (26). Sun et al. also found that the rate of lymphopenia was significantly lower in the hypofractionation group (16). This hypothesis can explain reduced RIL with hypofractionated radiation that RT kills less circulating lymphocytes, from less fractionation, thus less radiation exposure of the circulating lymphocytes (27). However, since $90.5 \%$ of our patients received hypofractionation radiation, we cannot validate the effect of fractionation. Further prospective validation with comparative distribution is needed to explore the real effect of hypofractionation radiation.

Prior chemotherapy appeared to be another risk factor for lymphopenia post-RT, while concurrent antiHER2 therapy and endocrine therapy were not. This is consistent with findings from a study by Sage et al. (28) 
and likely because of chemotherapy effect on bone marrow suppression $(29,30)$. However, chemotherapy was not an independent significant risk factor for nadir-PLC/pre-PLC in this study, implying that it had little influence on the ratio of PLC change during radiation, although it can decrease absolute PLC post-RT. Further detailed analysis is needed.

This study has limitations. First, as a retrospective study, it carries inherent flaws, such as missing data for PLC 1-3 months post-RT. However, this study selected consecutive patients from a single RT center and thus had relatively uniform treatment techniques and objective blood test measures. Second, there might have been selection bias for patients allocated to the 3DCRT and RapidArc groups, and our matched analysis may not have balanced all of the confounding factors. More extensive dosimetric studies are ongoing. Further studies are needed to validate our findings and investigate the effects and underlying mechanisms of RT techniques on the long-term survival of patients with breast cancer.

\section{Conclusions}

This study of 735 patients reported that RIL is common in breast cancer after adjuvant radiation, and the most important risk factors were RT factors, including RT technique and dosimetric factors. Our study demonstrated for the first time the importance of low dose lung volume associated with radiotherapy techniques like RapidArc on RIL in breast cancer, suggesting a need for further RT plan optimization in the future to minimize this toxicity and improve survival in patients.

\section{Acknowledgments}

Timothy S. K. Hui supported this work with language editing.

Funding: This work was supported in parts by the Health Commission of Guangdong Province, China [A2021114], Shenzhen Key Medical Discipline Construction Fund [SZXK014], and the Shenzhen Science and Technology program [KQTD20180411185028798].

\section{Footnote}

Reporting Checklist: The authors have completed the STROBE reporting checklist. Available at https://dx.doi. org/10.21037/atm-21-2150

Data Sharing Statement: The datasets analyzed during the current study are not publicly available due to privacy but are available from the corresponding author upon a reasonable request. Available at https://dx.doi.org/10.21037/ atm-21-2150

Conflicts of Interest: All authors have completed the ICMJE uniform disclosure form (available at https://dx.doi. org/10.21037/atm-21-2150). The authors have no conflicts of interest to declare.

Ethical Statement: The authors are accountable for all aspects of the work in ensuring that questions related to the accuracy or integrity of any part of the work are appropriately investigated and resolved. The study was conducted in accordance with the Declaration of Helsinki (as revised in 2013). The study was approved by the ethics committee of the University of Hong Kong-Shenzhen Hospital (\# 2019 098) and individual consent for this retrospective analysis was waived.

Open Access Statement: This is an Open Access article distributed in accordance with the Creative Commons Attribution-NonCommercial-NoDerivs 4.0 International License (CC BY-NC-ND 4.0), which permits the noncommercial replication and distribution of the article with the strict proviso that no changes or edits are made and the original work is properly cited (including links to both the formal publication through the relevant DOI and the license). See: https://creativecommons.org/licenses/by-nc-nd/4.0/.

\section{References}

1. Bray F, Ferlay J, Soerjomataram I, et al. Global cancer statistics 2018: GLOBOCAN estimates of incidence and mortality worldwide for 36 cancers in 185 countries. CA Cancer J Clin 2018;68:394-424.

2. Whelan TJ, Olivotto IA, Parulekar WR, et al. Regional Nodal Irradiation in Early-Stage Breast Cancer. N Engl J Med 2015;373:307-16.

3. Poortmans PM, Collette S, Kirkove C, et al. Internal Mammary and Medial Supraclavicular Irradiation in Breast Cancer. N Engl J Med 2015;373:317-27.

4. EBCTCG (Early Breast Cancer Trialists' Collaborative Group); McGale P, Taylor C, et al. Effect of radiotherapy after mastectomy and axillary surgery on 10-year recurrence and 20-year breast cancer mortality: metaanalysis of individual patient data for 8135 women in 22 randomised trials. Lancet 2014;383:2127-35. 
5. Campian JL, Ye X, Brock M, et al. Treatment-related lymphopenia in patients with stage III non-small-cell lung cancer. Cancer Invest 2013;31:183-8.

6. Manuel M, Tredan O, Bachelot T, et al. Lymphopenia combined with low TCR diversity (divpenia) predicts poor overall survival in metastatic breast cancer patients. Oncoimmunology 2012;1:432-40.

7. De Giorgi U, Mego M, Scarpi E, et al. Relationship between lymphocytopenia and circulating tumor cells as prognostic factors for overall survival in metastatic breast cancer. Clin Breast Cancer 2012;12:264-9.

8. Afghahi A, Purington N, Han SS, et al. Higher Absolute Lymphocyte Counts Predict Lower Mortality from EarlyStage Triple-Negative Breast Cancer. Clin Cancer Res 2018;24:2851-8.

9. Wild AT, Ye X, Ellsworth SG, et al. The Association Between Chemoradiation-related Lymphopenia and Clinical Outcomes in Patients With Locally Advanced Pancreatic Adenocarcinoma. Am J Clin Oncol 2015;38:259-65.

10. Tang C, Liao Z, Gomez D, et al. Lymphopenia association with gross tumor volume and lung V5 and its effects on non-small cell lung cancer patient outcomes. Int J Radiat Oncol Biol Phys 2014;89:1084-91.

11. Yovino S, Kleinberg L, Grossman SA, et al. The etiology of treatment-related lymphopenia in patients with malignant gliomas: modeling radiation dose to circulating lymphocytes explains clinical observations and suggests methods of modifying the impact of radiation on immune cells. Cancer Invest 2013;31:140-4.

12. Zhuang Y, Yuan BY, Chen GW, et al. Association Between Circulating Lymphocyte Populations and Outcome After Stereotactic Body Radiation Therapy in Patients With Hepatocellular Carcinoma. Front Oncol 2019;9:896.

13. So TH, Chan SK, Chan WL, et al. Lymphopenia and Radiation Dose to Circulating Lymphocytes With Neoadjuvant Chemoradiation in Esophageal Squamous Cell Carcinoma. Adv Radiat Oncol 2020;5:880-8.

14. Mehrazin R, Uzzo RG, Kutikov A, et al. Lymphopenia is an independent predictor of inferior outcome in papillary renal cell carcinoma. Urol Oncol 2015;33:388.e19-25.

15. Cho O, Chun M, Kim SW, et al. Lymphopenia as a Potential Predictor of Ipsilateral Breast Tumor Recurrence in Early Breast Cancer. Anticancer Res 2019;39:4467-74.

16. Sun GY, Wang SL, Song YW, et al. Radiation-Induced Lymphopenia Predicts Poorer Prognosis in Patients With Breast Cancer: A Post Hoc Analysis of a Randomized Controlled Trial of Postmastectomy Hypofractionated
Radiation Therapy. Int J Radiat Oncol Biol Phys 2020;108:277-85.

17. Diehl A, Yarchoan M, Hopkins A, et al. Relationships between lymphocyte counts and treatment-related toxicities and clinical responses in patients with solid tumors treated with PD-1 checkpoint inhibitors. Oncotarget 2017;8:114268-80.

18. Stjernswärd J, Jondal M, Vánky F, et al. Lymphopenia and change in distribution of human B and T lymphocytes in peripheral blood induced by irradiation for mammary carcinoma. Lancet 1972;1:1352-6.

19. Standish LJ, Torkelson C, Hamill FA, et al. Immune defects in breast cancer patients after radiotherapy. J Soc Integr Oncol 2008;6:110-21.

20. Ellsworth SG. Field size effects on the risk and severity of treatment-induced lymphopenia in patients undergoing radiation therapy for solid tumors. Adv Radiat Oncol 2018;3:512-9.

21. Nakamura N, Kusunoki Y, Akiyama M. Radiosensitivity of CD4 or CD8 positive human T-lymphocytes by an in vitro colony formation assay. Radiat Res 1990;123:224-7.

22. Hall EJ, Giaccia AJ. Radiobiology for the Radiologist. Lippincott Williams \& Wilkins; 2006.

23. Saito T, Toya R, Matsuyama T, et al. Dosimetric Predictors of Treatment-related Lymphopenia induced by Palliative Radiotherapy: Predictive Ability of Dose-volume Parameters based on Body Surface Contour. Radiol Oncol 2017;51:228-34.

24. Jin JY, Mereniuk T, Yalamanchali A, et al. A framework for modeling radiation induced lymphopenia in radiotherapy. Radiother Oncol 2020;144:105-13.

25. Crocenzi T, Cottam B, Newell P, et al. A hypofractionated radiation regimen avoids the lymphopenia associated with neoadjuvant chemoradiation therapy of borderline resectable and locally advanced pancreatic adenocarcinoma. J Immunother Cancer 2016;4:45.

26. Yuan C, Wang Q. Comparative analysis of the effect of different radiotherapy regimes on lymphocyte and its subpopulations in breast cancer patients. Clin Transl Oncol 2018;20:1219-25.

27. Jin J, Hu C, Xiao Y, et al. Higher radiation dose to immune system is correlated with poorer survival in patients with stage III non-small cell lung cancer: a secondary study of a phase 3 cooperative group trial (NRG Oncology RTOG 0617). Int J Radiat Oncol Biol Phys 2017;99:S151-2.

28. Sage EK, Schmid TE, Sedelmayr M, et al. Comparative analysis of the effects of radiotherapy versus radiotherapy after adjuvant chemotherapy on the composition of 
Page 14 of 14

lymphocyte subpopulations in breast cancer patients.

Radiother Oncol 2016;118:176-80.

29. Kotsakis A, Sarra E, Peraki M, et al. Docetaxel-induced lymphopenia in patients with solid tumors: a prospective phenotypic analysis. Cancer 2000;89:1380-6.

Cite this article as: Chen $\mathrm{F}, \mathrm{Yu} \mathrm{H}$, Zhang $\mathrm{H}$, Nong $\mathrm{Y}$, Wang Q, Jing H, Han Y, Wu J, Zhou Z, Yang L, Xu Z, Liu Y, Fu P, Jin JY, Hsue V, Chang A, Kong FM. Risk factors for radiation induced lymphopenia in patients with breast cancer receiving adjuvant radiotherapy. Ann Transl Med 2021;9(16):1288. doi: 10.21037/atm-21-2150
Chen et al. Radiation induced lymphopenia in breast cancer

30. Wijayahadi N, Haron MR, Stanslas J, et al. Changes in cellular immunity during chemotherapy for primary breast cancer with anthracycline regimens. J Chemother 2007;19:716-23. 